\title{
Capacity of OFDM Systems Over Fading Underwater Acoustic Channels
}

\author{
Chantri Polprasert, Member, IEEE, James A. Ritcey, Fellow, IEEE, and Milica Stojanovic, Fellow, IEEE
}

\begin{abstract}
In this paper, we derive bounds to the channel capacity of orthogonal frequency division multiplexing (OFDM) systems over the underwater (UW) acoustic fading channel as a function of the distance between the transmitter and the receiver. The upper bound is obtained under perfect channel state information (CSI) at the receiver. The lower bound is obtained assuming the input is drawn from phase-shift keying (PSK) constellation which results in non-Gaussian distribution of the output signal and no CSI. The reduction from the upper bound is due to limited mutual information that can be conveyed by PSK constellation and the linear minimum mean square prediction error. Our UW channel deviates from the wide sense stationary and uncorrelated scattering (WSSUS) model commonly used for small bandwidths. We incorporate frequency-dependent path loss due to the acoustic propagation into each arrival path between the transmitter and the receiver. This leads the UW channel to be modeled as a frequency-dependent doubly spread fading channel characterized by the wide sense stationary and correlated scattering (WSS-non-US) fading assumption. Both Rayleigh and Ricean fading assumptions are investigated in our model. Results from the model show a gap between the upper and lower bounds which depends not only on the ranges and shape of the scattering function of the UW channel but also on the distance between the transmitter and the receiver. Our model for the scattering function was suggested by Rescheduled Acoustic Communications Experiment (RACE08) experimental data, leading to a multilag autoregressive (AR-q) model for the fading.
\end{abstract}

Index Terms-Doubly spread fading channels, linear minimum mean square error, orthogonal frequency division multiplexing (OFDM), underwater acoustic communications, underwater acoustic propagation, wideband channel capacity.

\section{INTRODUCTION}

$\mathbf{O}$ RTHOGONAL FREQUENCY DIVISION MULTIPLEXING (OFDM) [1] is a multicarrier transmission technique which has been widely used in many broadband wireless standards such as digital audio/video coding (DAB/DVB), wireless local area network (IEEE802.11g), and WiMax (IEEE802.16). OFDM avoids intersymbol interference (ISI)

Manuscript received January 26, 2009; revised July 17, 2010 and April 04, 2011; accepted August 26, 2011. Date of publication September 29, 2011; date of current version October 21, 2011. This paper was supported by the U.S. Office of Naval Research under Grants N00014-07-1-0600 and N00014-07-1-0738 and the National Science Foundation under Grant 0831728.

Associate Editor: U. Mitra.

C. Polprasert is with the National Electronics and Computer Technology Center, Wireless Innovation and Security Lab, Pathumthani 12120, Thailand (e-mail: chantri.polprasert@nectec.or.th).

J. A. Ritcey is with the Electrical Engineering Department, University of Washington, Seattle, WA 98195-2500 USA (e-mail: ritcey@ee.washington.edu).

M. Stojanovic is with the Electrical and Computer Engineering Department, Northeastern University, Boston, MA 02115 USA (e-mail: millitsa@ece.neu. edu).

Digital Object Identifier 10.1109/JOE.2011.2167071 from multipath fading by transmitting data in parallel subchannels with gains equal to the channel's frequency response. Recently, OFDM has been applied to the underwater (UW) acoustic communications [2]-[5] and yields high data rate with strong bit error rate performance.

Data transmissions through UW channels prove challenging due to the detrimental effect of time and frequency spreading. Time spreading is caused by not only the arrival of multiple delay paths, but also the channel physics such as the attenuation and reflection loss which induce frequency-dependent path loss on each path [6], [7]. For frequency spreading, relative movement between the transmitters and the receivers or dynamic motion of the medium causes time-varying Doppler shift which can be significant due to the slow sound speed. Several attempts have been made for realistic channel characterization and the widely accepted one views the UW channel as a linear time-varying channel with wide sense stationary and uncorrelated scattering (WSSUS) [8]-[10]. However, this treats the entire frequency band as a whole, neglecting frequency-dependent path loss due to the channel physics on each arrival path. This model is acceptable for data transmission only at low bandwidth $(<10 \mathrm{kHz})$ [9].

Channel capacity [11] has been used as a benchmark for determining the maximum data rate given a source power and bandwidth. To exhibit the capacity under practical limitations, several works [12]-[15] study the capacity over WSSUS fading channels under the following assumptions: 1) no channel state information (CSI) is available at the transmitter or the receiver, and 2) peak power constraints. It is shown that channel capacity is achieved at a specific signal bandwidth, called the capacity maximizing bandwidth, which depends on the ranges and shape of the scattering function of the fading channel. These studies have been conducted over wireless fading channels which assume constant power spectral density (PSD) and additive white Gaussian noise (AWGN).

Despite widespread research on the capacity for wireless communications, few have been done in the UW acoustic communications. Kwon and Birdsal [16] study the capacity over a time-invariant channel with additive Gaussian noise. Hayward and Yang [17] analyze the capacity over single and multiple channels using data from experiments. Stojanovic [6] develops the capacity as a function of the transmission distance between the transmitter and the receiver. All assume no fading in their UW channels.

In this paper, influenced by [12]-[15], we investigate the capacity of OFDM systems over the UW fading channels with no CSI at the transmitter or the receiver. The main difference of our work from [12]-[15] is the characterization of the UW channel. The UW channel is modeled by taking into account frequency- 
dependent path loss due to the acoustic propagation which depends on the signal bandwidth and propagation distance of each arrival path. This invalidates the assumption of stationarity in frequency of the WSSUS model and leads to a frequency-dependent doubly spread (DS) fading channels characterized by the wide sense stationary and correlated scattering (WSS-non-US) [18] assumptions. Compared to the conventional WSSUS model which is uncorrelated in both delay and Doppler domains, the proposed model is still uncorrelated in the Doppler but correlated in the delay domain. Using this channel model, we derive the capacity upper and lower bounds by assuming the acoustic propagation and ambient noise PSD are available at both the transmitter and the receiver. The capacity upper bound is derived assuming perfect CSI at the receiver. The capacity lower bound is obtained by the mutual information rate whose input is an independent and identically distribution (i.i.d.) random variable (RV) and is drawn from a PSK modulation [12], [19]. This results in a non-Gaussian distribution for the output signal. Based on several fading models of the underwater channels [8], results are obtained when statistical property of the UW channels is model by Rayleigh and Ricean fading. Reduction from the upper bound is due to the limited mutual information that can be conveyed by PSK constellation and the linear minimum mean square error (MMSE) prediction error. Simulation results show a gap between the upper and lower bounds which depends not only on the ranges and shape of the scattering function of the UW channel, but also the distance between the transmitter and the receiver. Results are confirmed when tested with the scattering function we obtain from the 2008 Rescheduled Acoustic Communications Experiment (RACE08) experimental data.

We use cyclic-prefix OFDM (CP-OFDM) as a framework to investigate channel capacity. Applications to other block-based multicarrier transmissions are straightforward and will be considered as future work.

This paper is organized as follows. In Section II, we discuss OFDM system model and propose frequency-dependent UW DS fading channels. Then, we derive capacity upper and lower bounds of the OFDM systems in Section III. ICI analysis on the limitations of our model and simulation results are presented in Section IV. Section V shows results over experimental data.

Notation: Let $\boldsymbol{x}$ be a column vector. $\operatorname{diag}(\boldsymbol{x})$ is a diagonal matrix with $\boldsymbol{x}$ on its diagonal; $E[\cdot]$ is the expectation; $(\cdot)^{*},(\cdot)^{H}$, $(\cdot)^{T}$ denote complex conjugate, Hermitian, and transpose, respectively; $\boldsymbol{x} \sim \mathcal{C N}\left(\mathbf{0}, \boldsymbol{R}_{\boldsymbol{x}}\right)$ means a circularly symmetric complex Gaussian random vector with zero mean and covariance matrix $E\left[\boldsymbol{x} \boldsymbol{x}^{H}\right]=\boldsymbol{R}_{\boldsymbol{x}} ; \operatorname{sinc}(x)=\sin (\pi x) /(\pi x) ; \otimes$ is convolution. $A \odot B$ is the element-wise product of matrix $A$ and $B$.

\section{OFDM SYSTEM AND UW CHANNEL MODEL}

In this section, we develop an OFDM system model for UW acoustic communications. Then, we study physical and statistical properties of UW channels and propose a frequency-dependent UW DS fading channel. Finally, the PSD of the ambient noise is investigated.

\section{A. OFDM System}

We consider a conventional CP-OFDM system [1]. The discrete-time equivalent baseband model is shown in Fig. 1.

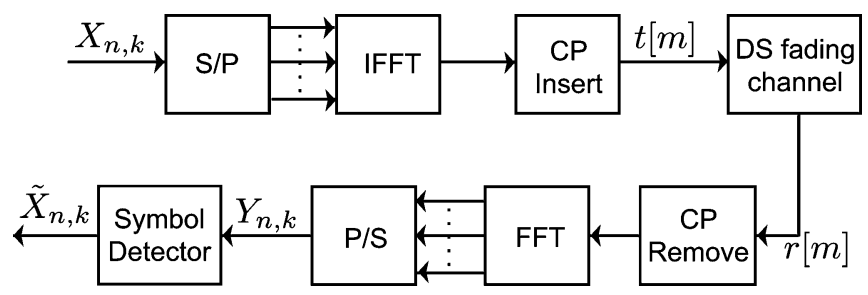

Fig. 1. System model.

Let $\mathbf{X}_{n}=\left[X_{n, 0} \cdots X_{n, K-1}\right]^{T}$ and $\mathbf{Y}_{n}=$ $\left[Y_{n, 0} \cdots Y_{n, K-1}\right]^{T}$ denote an input and its corresponding output block of data which are sent and received at the $n$th OFDM symbol duration, respectively. Assuming the guard interval $L_{c p}$ is longer than the channel length $L$ to avoid the interblock interference (IBI), the input/output relationship can be written as

$$
Y_{n, k}=G_{n, k}(d) X_{n, k}+N_{n, k}
$$

where $k \in[0, \ldots, K-1]$ is the subcarrier index and $n \in$ $[0, N-1] . d$ is the distance between the transmitter and the receiver. $G_{n, k}(d)$ denotes the channel transfer function at the $k$ th subcarrier. This simplifies the fading effect into multiplicative coefficient and will be the basis for analysis of the UW channels for the rest of this paper. Its relationship with the distance $d$ will be elaborated on in Section II-B. We assume the impact of ICI is negligible through appropriate parameter settings discussed in Section IV-A and justified in Appendix I. $N_{n, k}$ is the ambient noise in the ocean explained in Section II-C.

For simplicity, we characterize the overall system input/output of the entire $N$ OFDM transmissions by a vector of size $N K \times 1$

$$
\mathbf{Y}=\operatorname{diag}(\mathbf{X}) \mathbf{G}(d)+\mathbf{N}=\operatorname{diag}(\mathbf{G}(d)) \mathbf{X}+\mathbf{N}
$$

where

$$
\begin{aligned}
& \mathbf{Y}=\left[\mathbf{Y}_{0}^{T} \ldots \mathbf{Y}_{N-1}^{T}\right]^{T} \quad \text { and } \quad \mathbf{Y}_{n}=\left[Y_{n, 0} \ldots Y_{n, K-1}\right]^{T} \\
& \mathbf{X}=\left[\mathbf{X}_{0}^{T} \ldots \mathbf{X}_{N-1}^{T}\right]^{T} \text { and } \mathbf{X}_{n}=\left[X_{n, 0} \ldots X_{n, K-1}\right]^{T} \\
& \mathbf{N}=\left[\mathbf{N}_{0}^{T} \ldots \mathbf{N}_{N-1}^{T}\right]^{T} \quad \text { and } \quad \mathbf{N}_{n}=\left[N_{n, 0} \ldots N_{n, K-1}\right]^{T}
\end{aligned}
$$$$
\mathbf{G}(d)=\left[\mathbf{G}_{0}^{T}(d) \ldots \mathbf{G}_{N-1}^{T}(d)\right]^{T}
$$

and

$\mathbf{G}_{n}(d)=\left[G_{n, 0}(d) \ldots G_{n, K-1}(d)\right]^{T}$.

These matrices will be used to derive channel capacity in Section III.

\section{B. Characterization of the Approximate DS Fading Channels}

UW channels are characterized by physical and statistical properties. The physical property is the attenuation which depends on the propagation distance and bandwidth of the transmitted signal. For the statistical part, the channel is usually assumed WSSUS. We model a channel by taking into account both properties to form a frequency-dependent DS fading channel. 
1) Frequency-Dependent Path Loss: For signals propagated through UW medium, its energy is attenuated as a function of both the distance and signal frequency. This attenuation or path loss is a combination of the geometric spreading and absorption and can be written as [23]

$$
Q^{2}(d, f)=d^{-s p}\left(q^{2}(f)\right)^{-d}
$$

where $d$ is the distance (meter) the signal has propagated and $f$ is the frequency in kilohertz. $d^{-s p}$ represents the spreading loss and $s p$ is the spreading factor which is set to $1.5 . q^{2}(f)$ is the absorption coefficient in seawater which depends on numerous parameters and is given by [23]

$$
\begin{aligned}
10 \log \left(q^{2}(f)\right)=2.49 \times & 10^{-7} f^{2}+0.99 \frac{f^{2}}{f^{2}+1.23 \times 10^{4}} \\
& +1.48 \times 10^{-4} \frac{f^{2}}{f^{2}+1.522} \mathrm{~dB} / \mathrm{m}
\end{aligned}
$$

Equation (8) is calculated when the salinity $S$ is 35 parts per thousand (ppt), gauge pressure $P_{a}$ is $1 \mathrm{~atm}$, temperature $T=14$ ${ }^{\circ} \mathrm{C}$, and the relaxation frequency is $111 \mathrm{kHz}$. These parameters are used for the rest of this paper.

2) Conventional Statistical Model: In [9] and [10], UW CIR is modeled by a sum of several multipath components. Let $h(t, \tau)$ denote a continuous-time CIR of linear time-variant (LTV) UW channels and its corresponding transfer function $H(t, f)$

$$
h(t, \tau)=\sum_{i=0}^{I-1} h_{i}(t) \delta\left(\tau-\tau_{i}\right), \quad H(t, f)=\sum_{i=0}^{I-1} h_{i}(t) e^{-j 2 \pi f \tau_{i}}
$$

where $I$ is the number of arrival paths. WSSUS is commonly assumed to characterize the channel [8]-[10], [24], i.e., $E\left\{h[t, \tau] h^{*}\left[t^{\prime}, \tau^{\prime}\right]\right\}=R_{h_{c}}\left(t-t^{\prime}, \tau\right) \delta\left(\tau-\tau^{\prime}\right)$ where $R_{h_{c}}\left(t-t^{\prime}, \tau\right)$ is the autocorrelation function of the delay $\tau$ between time $t$ and $t^{\prime}$. Its corresponding scattering function is $S_{c}(\tau, \nu)=\int R_{h_{c}}(\Delta t, \tau) \exp (-j 2 \pi \Delta t \nu) d \Delta t$ where $\tau \in\left[0, \tau_{m}\right]$. Let $\tau_{m}$ and $f_{d}$ denote the maximum channel delay spread and 3-dB Doppler spread of $S_{c}(\tau, \nu)$, respectively. This assumption is appropriate when the bandwidth is less than 10 $\mathrm{kHz}$ [9].

3) Frequency-Dependent DS Fading Channels: Conventional UW propagation studies use WSSUS properties to characterize LTV UW channels, assuming equal attenuation across the signal bandwidth. This treats the entire frequency band as flat, neglecting frequency-dependent parameters of the individual arrival path. Frequency dependence of the instantaneous channel transfer function comes from different arrival time of the delay paths. However, various factors from channel physics such as the attenuation, reflection loss, or the transmitter/receiver operating ranges influence frequency dependency on the path loss. In this paper, we limit the impact of channel physics to only the attenuation $Q^{2}\left(d_{i}, f\right)(7)$ where $d_{i}$ is the propagation distance of the $i$ th delay path. Let $\chi_{d_{i}}(\tau)$ denote a CIR of the $i$ th delay path corresponding to $Q\left(d_{i}, f\right)$, i.e., $Q\left(d_{i}, f\right)=\int \chi_{d_{i}}(\tau) \exp (-j 2 \pi f \tau) d \tau$ where
$Q^{2}\left(d_{i}, f\right)=Q\left(d_{i}, f\right) Q^{*}\left(d_{i}, f\right)$. Taking into account $\chi_{d_{i}}(\tau)$ yields a modified channel CIR, $g_{d}(t, \tau)$

$$
\begin{aligned}
g_{d}(t, \tau) & =\sum_{i=0}^{I-1} h_{i}(t) \chi_{d_{i}}(\tau) \otimes \delta\left(\tau-\tau_{i}\right) \\
G_{d}(t, f) & =\int g_{d}(t, \tau) e^{-j 2 \pi \tau f} d \tau \\
& =\sum_{i=0}^{I-1} h_{i}(t) Q\left(d_{i}, f\right) e^{-j 2 \pi \tau_{i} f} \\
& \approx Q\left(d_{0}, f\right) \sum_{i=0}^{I-1} h_{i}(t) e^{-j 2 \pi \tau_{i} f} \\
& =Q(d, f) H(t, f) .
\end{aligned}
$$

$d_{0}$ is the distance between the transmitter and the receiver and we neglect the subscript of $d$ in (13) for simplicity. The approximation (12) is assumed given that $Q(d, f) \approx Q\left(d_{I-1}, f\right)$. This is justified when $\tau_{I-1}-\tau_{0}<10 \mathrm{~ms}$. $\left(\left|Q\left(d_{I-1}, f\right)\right|\right.$ is within $2.62 \%$ of $|Q(d, f)|$ for $f<50 \mathrm{kHz}$ and $d \geq 5 \mathrm{~km}$.) Hence, the modified CIR is

$$
g_{d}(t, \tau)=\chi_{d}(\tau) \otimes \sum_{i=0}^{I-1} h_{i}(t) \delta\left(\tau-\tau_{i}\right)
$$

From the sampling theorem, the $T_{s}$-spaced discrete-time CIR is [25]

$$
\begin{aligned}
g_{0, d}\left[m, p_{l}\right] & =\int_{\tau} g_{d}\left(m T_{s}, \tau\right) \operatorname{sinc}\left(B \tau-p_{l}\right) d \tau \\
& \approx g_{d}\left(m T_{s}, p_{l} T_{s}\right) \\
& =\chi_{d}[l] \otimes h_{0}\left[m, p_{l}\right]
\end{aligned}
$$

where $B=1 / T_{s}$ and the approximation (16) is assumed for large $B . \chi_{d}[l] \approx \chi_{d}\left(l T_{s}\right)$ and $h_{0}\left[m, p_{l}\right] \approx h\left(m T_{s}, p_{l} T_{s}\right) . l \in$ $[0, \ldots, L-1], L=\left\lceil\tau_{m} / T_{s}\right\rceil$ and $\tau_{m}=\tau_{I-1}$ is the maximum channel delay spread. From (17), the channel transfer function $G_{n, k}(d)$ can be written as

$$
\begin{aligned}
G_{n, k}(d) & \sum_{m^{\prime}=0}^{K-1}\left(\frac{1}{K} \sum_{l=0}^{L-1} g_{0, d}^{n}\left[m^{\prime}+L_{c p}, l\right] \sum_{p=0}^{K-1} e^{j 2 \pi p\left(m^{\prime}-l\right) / K}\right) \\
& \times e^{-j 2 \pi m^{\prime} k / K} \\
= & \sum_{p=0}^{K-1} \sum_{l=0}^{L-1}\left(\frac{1}{K} \sum_{m^{\prime}=0}^{K-1}\left(\chi_{d}[l] \otimes h_{0}^{n}\left[m^{\prime}+L_{c p}, l\right]\right)\right. \\
= & \left.\sum_{p=0}^{K-1} \sum_{l=0}^{L-1} e^{j 2 \pi m^{\prime}(p-k) / K}\right) e^{-j 2 \pi p l / K} \\
\approx & \sum_{l=0}^{L-1} G_{n, l}^{d}[0] e^{-j 2 \pi k l / K} \\
= & Q\left(d, f_{k}\right) H_{n, k}
\end{aligned}
$$


where $f_{k}=f_{c}+k /\left(K T_{s}\right)$ and $f_{c}$ is the center frequency corresponding to the zeroth subcarrier. $g_{0, d}^{n}\left[m^{\prime}, l\right]=g_{0, d}\left[n N_{s}+\right.$ $\left.p_{0}+m^{\prime}, p_{l}\right]$ and $h^{n}\left[m^{\prime}, l\right]=h_{0}\left[n N_{s}+p_{0}+m^{\prime}, p_{l}\right]$ where $N_{s}=L_{c p}+K$ is the OFDM symbol length and $p_{0}$ is the arrival time of the first arrival path. Equation (21) is obtained under negligible ICI which will be discussed in Section IV. Moreover

$$
\begin{aligned}
H_{n, k} & =\sum_{l=0}^{L-1}\left(\frac{1}{K} \sum_{m^{\prime}=0}^{K-1} h_{0}^{n}\left[m^{\prime}+L_{c p}, l\right]\right) e^{-j 2 \pi l k / K} \\
& =\sum_{l=0}^{L-1} h_{n, l}[0] e^{-j 2 \pi l k / K} \\
Q\left(d, f_{k}\right) & =\sum_{l=0}^{L-1} \chi_{d}[l] e^{-j 2 \pi l k / K}
\end{aligned}
$$

and

$G_{n, l}^{d}[p-k]=\frac{1}{K} \sum_{m^{\prime}=0}^{K-1}\left(\chi_{d}[l] \otimes h_{0}^{n}\left[m^{\prime}+L_{c p}, l\right]\right) e^{j 2 \pi m^{\prime}(p-k) / K}$.

$G_{n, k}(d)$ is the fading gain encountered by the signal transmitted on the $k$ th subcarrier. $Q\left(d, f_{k}\right)$ is assumed constant within a subcarrier with center frequency $f_{k} . h_{n, k}[0]$ is the approximate CIR. Expression (22) simplifies the transfer function of the frequency-dependent UW DS channels into a multiplication of two terms: the attenuation $Q\left(d, f_{k}\right)$ and statistical $H_{n, k}$ parts governed by the scattering function $S[l, \lambda]$. We assume $S[l, \lambda] \approx$ $S_{c}\left(l T_{s}, \lambda / T_{b}\right)$ when the variation of $h_{0}\left[m, p_{l}\right]$ within $T_{b}\left(T_{b}=\right.$ $\left.N_{s} T_{s}\right)$ is negligible [32]. $T_{b}$ is the OFDM symbol interval $\lambda \in$ $[-0.5,0.5]$. Its range $\left(L, \lambda_{d}\right)$ is related to $\left(\tau, f_{d}\right)$ of $S_{c}(\tau, \nu)$ through $L=\left\lceil\tau_{m} B\right\rceil$ and $\lambda_{d}=f_{d} T_{b}$. This leads $G_{n, k}(d)$ to a WSS but non-US fading channel [18]

$$
\begin{aligned}
& E\left\{G_{n^{\prime}, k^{\prime}}(d) G_{n, k}^{*}(d)\right\} \\
& \quad=Q\left(d, f_{k}\right) Q^{*}\left(d, f_{k^{\prime}}\right) R_{H}\left[n-n^{\prime}, k-k^{\prime}\right]
\end{aligned}
$$

where $R_{H}\left[n-n^{\prime}, k-k^{\prime}\right]=E\left[H_{n, k} H_{n^{\prime}, k^{\prime}}^{*}\right]$. Compared to the conventional WSSUS model which is uncorrelated in both delay and Doppler domains due to the stationarity in time and frequency, the proposed model $G_{n, k}(d)$ is still uncorrelated in Doppler but correlated in the delay domain because of the attenuation. To further investigate $G_{n, k}(d)$, Fig. 2 shows a realization of $\left|G_{n, k}(d)\right|^{2}$ when $d=5$ and $20 \mathrm{~km}$. The approximate $\operatorname{CIR} h_{n, l}[0]$, which generates the statistical part $H_{n, k}$, is assumed zero-mean complex Gaussian random variables with exponentially decaying power delay profile (PDP) with 20-dB power difference between the first and last paths. Transmit bandwidth is $51.2 \mathrm{kHz}$. Channel delay length is assumed $5 \mathrm{~ms}$ which corresponds to $L=256$. The number of subcarriers $K$ is 512. From Fig. 2, it is evident that the propagation distance and signal frequency have a significant impact on the realization of $\left|G_{n, k}(d)\right|^{2}$.

To be consistent with (2)-(5), from (22), a vector form for $G_{n, k}(d)$ is

$$
\mathbf{G}_{n}(d)=\mathbf{Q}(d) \mathbf{H}_{n}
$$

where $\mathbf{Q}(d)=\operatorname{diag}\left(\left[Q\left(d, f_{0}\right) \cdots Q\left(d, f_{K-1}\right)\right]\right)$ and $\mathbf{H}_{n}=$ $\left[H_{n, 0} \cdots H_{n, K-1}\right]^{T}$ from (23).

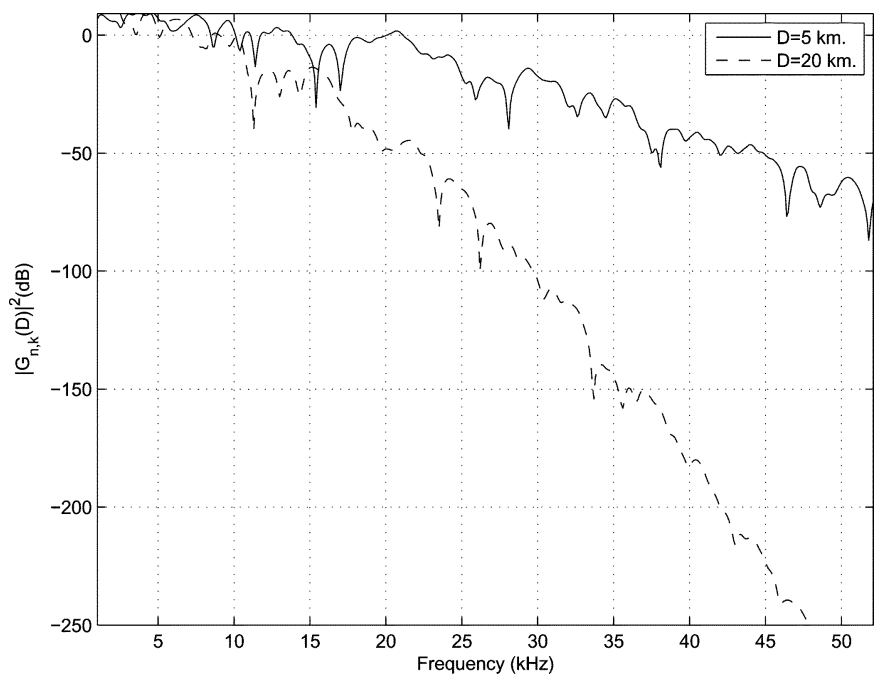

Fig. 2. Impact of the attenuation $Q^{2}\left(d, f_{k}\right)$ on a realization of $\left|G_{n, k}(d)\right|^{2}$ when for $d=5$ and $20 \mathrm{~km}$.

\section{Ambient Noise}

$N_{n, k}$ in (1) is assumed the ambient noise in the ocean. The ambient noise consists of four sources [6]: turbulence $A_{t}(f)$, shipping $A_{s}(f)$, waves $A_{w}(f)$, and thermal noise $A_{t h}(f)$. They can be described by Gaussian statistics with a continuous PSD in $\mathrm{dBre} / \mu \mathrm{Pa}$ per hertz

$$
\begin{aligned}
& A_{t}(f)=17-30 \log f \\
& A_{s}(f)=40+20(s-0.5)+26 \log f-60 \log (f+0.03) \\
& A_{w}(f)=50+7.5 \sqrt{w}+20 \log f-40 \log (f+0.4) \\
& A_{t h}(f)=-15+20 \log f
\end{aligned}
$$

where $f$ is frequency in kilohertz and the overall noise PSD is

$$
\begin{aligned}
A(f)=10 \log \left(10^{A_{t}(f) / 10}+10^{A_{s}(f) / 10}\right. & \\
+ & \left.10^{A_{w}(f) / 10}+10^{A_{t h}(f) / 10}\right) .
\end{aligned}
$$

$s \in[0,1]$ is the shipping activity and $w$ is the wind speed in meters per second. The ratio of the attenuation $Q^{2}(d, f)$ and the ambient noise PSD $A(f)$ is crucial in determining a frequency band that maximizes signal-to-noise ratio (SNR) for each transmission distance.

\section{CAPACITY OF THE UW CHANNELS}

We derive the upper bound $U_{c}(d)$ and the lower bound $L_{c}(d)$ of the ergodic channel capacity of OFDM systems over UW fading channels as a function of the distance $d$ between the transmitter and the receiver. The capacity $C(d)$ is given in bits per second by

$$
C(d)=\lim _{N \rightarrow \infty} \frac{1}{N T_{b}} \sup _{p(\mathbf{X})} I(\mathbf{Y} ; \mathbf{X}) .
$$

$\mathbf{X}$ and $\mathbf{Y}$ are the transmitted and received vectors as defined in (2)-(4) and the maximization is over the set $p(\mathbf{X})$ of all input distributions that satisfy a given average-power constraint. $T_{b}$ is OFDM symbol interval $\left(T_{b}=N_{s} T_{s}\right) . U_{c}(d)$ is obtained when the input vector follows a joint complex Gaussian distribution. $L_{c}(d)$ is obtained under imperfect CSI whose reduction from $U_{c}(d)$ comes from limited mutual information from PSK constellation and the MMSE prediction error related to channel uncertainty [12], [14]. This bounding technique is used 
in [12] for wireless fading channels. Ours is for UW channels, which exhibit different results because of the frequency-dependent fading statistics of the channels.

The bounds are derived under the following assumptions.

- Information of the attenuation $Q^{2}(d, f)(7)$ and the ambient noise PSD $A(f)(29)$ of the UW channels are available at both the transmitter and the receiver.

- For the statistical part $\left[H_{n, k}(23)\right]$ of UW channels, its approximate CIR $h_{n, l}[0]$ is assumed a WSSUS random process with variance $\sigma_{l}^{2}$ where $\sum_{l} \sigma_{l}^{2}=E\left[\left|H_{n, k}\right|^{2}\right]=1$. Rayleigh and Ricean fading are assumed in Sections III-B and III-C, respectively. A scattering function which characterizes $H_{n, k}$ is available at the receiver.

- The noise vector $\mathbf{N} \sim \mathcal{C N}(\mathbf{0}, \operatorname{diag}(\mathbf{A})) . \mathbf{A}=$ $\left[\mathbf{A}_{0} \ldots \mathbf{A}_{N-1}\right]^{T}$ and $\mathbf{A}_{n}=\left[A\left(f_{0}\right), \ldots, A\left(f_{K-1}\right)\right]^{T}$ from (29).

- The impact of the ICI is negligible compared to $A\left(f_{k}\right)$.

Let $F$ denote the subcarrier spacing and $B=K F$ the signal bandwidth. $P$ is the signal transmit power in $\mathrm{dBre} / \mu \mathrm{Pa}$.

\section{A. Upper Bound $U_{c}(d)$}

To bound $\sup _{p(\mathbf{X})} I(\mathbf{Y} ; \mathbf{X})$, we use the chain rule $I(\mathbf{Y} ; \mathbf{X})=I(\mathbf{Y} ; \mathbf{X}, \mathbf{G}(d))-I(\mathbf{Y} ; \mathbf{G}(d) \mid \mathbf{X})$. The output vector $\mathbf{Y}$ depends on the input vector $\mathbf{X}$ through $\mathbf{b}=$ $\operatorname{diag}(\mathbf{X}) \mathbf{G}(d)$, so $I(\mathbf{Y} ; \mathbf{X}, \mathbf{G}(d))=I(\mathbf{Y} ; \mathbf{b})$. The upper bound of $I(\mathbf{Y} ; \mathbf{b})$ is achieved when the input $\mathbf{b} \sim$ $\mathcal{C N}\left(\mathbf{0}, \mathbf{I}+\mathbf{R}_{\mathbf{X}}(d) \odot \mathbf{R}_{\mathbf{G}}(d)\right) . \mathbf{R}_{\mathbf{G}}(d)=\mathrm{E}\left[\mathbf{G}(d) \mathbf{G}^{H}(d)\right]$. $\mathbf{R}_{\mathbf{X}}(d)=\operatorname{diag}\left(\left[\mathbf{R}_{\mathbf{X}_{\mathbf{0}}}(d), \ldots, \mathbf{R}_{\mathbf{X}_{\mathbf{N}-1}}(d)\right]\right)$ where $\mathbf{R}_{\mathbf{X}_{\mathbf{n}}}(d)=$ $\operatorname{diag}\left(\left[\sigma_{x}^{2}\left(d, f_{0}\right) \ldots \sigma_{x}^{2}\left(d, f_{K}-1\right)\right]\right)$ and $\sigma_{x}^{2}\left(d, f_{k}\right)=$ $E\left[\left|X_{n, k}\right|^{2}\right]$. The upper bound $U_{c}(d)$ is [27]

$$
\begin{aligned}
& C(d) \leq \lim _{N \rightarrow \infty} \frac{1}{N T_{b}} \sup _{\mathbf{R}_{\mathbf{X}}(d)} \log \operatorname{det}\left(\mathbf{I}+\left(\mathbf{R}_{\mathbf{X}}(d) \odot \mathbf{R}_{\mathbf{G}}(d)\right)\right. \\
&\left.\times \operatorname{diag}(\mathbf{A})^{-1}\right) \\
& \leq \lim _{N \rightarrow \infty} \frac{1}{N T_{b}} \sup _{\mathbf{R}_{\mathbf{X}}(d)} \sum_{n=0}^{N-1} \sum_{k=0}^{K-1} \log \left(1+E\left[\left|X_{n, k}\right|^{2}\right]\right. \\
&\left.\times \frac{Q^{2}\left(d, f_{k}\right)}{A\left(f_{k}\right)}\right) \\
&=\frac{1}{T_{b}} \sum_{k=0}^{K-1} \sup _{\sigma_{x}^{2}\left(d, f_{k}\right)} \log \left(1+\sigma_{x}^{2}\left(d, f_{k}\right) \frac{Q^{2}\left(d, f_{k}\right)}{A\left(f_{k}\right)}\right) \\
&=U_{c}(d) .
\end{aligned}
$$

Inequality (31) follows from Hadamard's inequality [11]. This result is similar to [6] which is the capacity of the time-invariant UW channels but ours is scaled by a factor of $F T_{b}$ which is greater than one to avoid the IBI. $\sigma_{x}^{2}\left(d, f_{k}\right)$ is subject to the source power constraint

$$
F \sum_{k=0}^{K-1} \sigma_{x}^{2}\left(d, f_{k}\right)=P
$$

$U_{c}$ is obtained when energy allocation across subcarriers satisfies

$$
\sigma_{x}^{2}\left(d, f_{k}\right)= \begin{cases}{\left[T_{h}-\left(\frac{A\left(f_{k}\right)}{Q\left(d, f_{k}\right)^{2}}\right)\right]^{+},} & f_{k} \in B \\ 0, & \text { else }\end{cases}
$$

where $T_{h}$ is chosen so that (33) is satisfied according to the water-filling algorithm $[11]$ and $[x]^{+}$is defined as $\max (x, 0)$.

\section{B. Lower Bound $L_{c}(d)$ Over Rayleigh Fading Channels}

We lower bound the capacity (30) by the mutual information rate $I(\mathbf{Y} ; \mathbf{X}) / N T_{b}$ when $\mathbf{X}$ is drawn from a PSK constellation. Channel fading statistics are assumed available at the receiver, not the transmitter. We follow a similar approach [12] to determine $L_{c}(d)$ but our results show that the decrease in $L_{c}(d)$ depends not only on the channel variations but also on the propagation distance $d$ between the transmitter and the receiver. This result has never been presented anywhere for UW channels.

Consider the mutual information $I(\mathbf{Y} ; \mathbf{X})$ where each entry of $\mathbf{X}, X_{n, k}$, is drawn from PSK modulation whose amplitude $\left|X_{n, k}\right|=\sigma_{x}$ and phase $\angle X_{n, k}$ has a uniform discrete distribution across a circle. $X_{n, k}$ is an i.i.d. RV. Using the chain rule, $I(\mathbf{Y} ; \mathbf{X})$ can be written as

$$
\begin{aligned}
I(\mathbf{Y} ; \mathbf{X}) & =I(\mathbf{Y} ; \mathbf{X}, \mathbf{G}(d))-I(\mathbf{Y} ; \mathbf{G}(d) \mid \mathbf{X}) \\
& \geq I(\mathbf{Y} ; \mathbf{X} \mid \mathbf{G}(d))-I(\mathbf{Y} ; \mathbf{G}(d) \mid \mathbf{X})
\end{aligned}
$$

The inequality in (36) is due to the nonnegativity property of the mutual information. Exact calculation of the mutual information is infeasible due to the non-Gaussian distribution of $\mathbf{Y}$ [28]. Note that [29]

$$
I(\mathbf{Y} ; \mathbf{X} \mid \mathbf{G}(d))=N I\left(\mathbf{Y}_{N} ; \mathbf{X}_{N} \mid \mathbf{G}_{N}(d)\right)
$$

where $\mathbf{G}_{N}(d)=\mathbf{G}_{n}(d), \mathbf{X}_{N}=\mathbf{X}_{n}$, and $\mathbf{Y}_{N}=\mathbf{Y}_{n}$ since the input $X_{n, k}$ has an i.i.d. distribution and every block of the channel coefficients $\mathbf{G}_{n}(d)$ has the same distribution. We set $\sigma_{x}^{2}\left(d, f_{k}\right)$ according to (34) under constraint (33) and apply it to $I\left(\mathbf{Y}_{N} ; \mathbf{X}_{N} \mid \mathbf{G}_{N}(d)\right)$. This water-filling policy is suboptimal for PSK constellation [30].

$I(\mathbf{Y} ; \mathbf{G}(d) \mid \mathbf{X})$ is calculated in Appendix II which yields

$$
I(\mathbf{Y} ; \mathbf{G}(d) \mid \mathbf{X})=\sum_{n=0}^{N-1} \log \operatorname{det}\left(\boldsymbol{I}+\mathbf{B}_{n}(d) \operatorname{diag}(\mathbf{S}(d))\right)
$$

where $\mathbf{S}(d)$ is the $K \times 1$ vector whose $k$ th entry is $\sigma_{x}^{2}\left(d, f_{k}\right) Q^{2}\left(d, f_{k}\right) / A\left(f_{k}\right) . \mathbf{B}_{n}(d)$ is the linear MMSE prediction error matrix which depends on both the transmission distance $d$ and channel variation $R_{H}[m, k]$. Then, substituting (37) and (38) into (36), the mutual information $I(\mathbf{X} ; \mathbf{Y})$ is

$$
\begin{aligned}
I(\mathbf{X} ; \mathbf{Y}) \geq N I\left(\mathbf{Y}_{N} ; \mathbf{X}_{N} \mid \mathbf{G}_{N}(d)\right) & \\
& -\sum_{n=0}^{N-1} \log \operatorname{det}\left(\boldsymbol{I}+\mathbf{B}_{n}(d) \operatorname{diag}(\mathbf{S}(d))\right) .
\end{aligned}
$$

Finally, the lower bound $L_{c}(d)$ of the capacity $C(d)$ can be written as

$$
\begin{aligned}
C(d) \geq & \lim _{N \rightarrow \infty} \frac{1}{N T_{b}} I(\mathbf{Y} ; \mathbf{X}) \\
\geq & \frac{1}{T_{b}} I\left(\mathbf{Y}_{\infty} ; \mathbf{X}_{\infty} \mid \mathbf{G}_{\infty}(d)\right) \\
& -\frac{1}{T_{b}} \log \operatorname{det}\left(\boldsymbol{I}+\mathbf{B}_{\infty}(d) \operatorname{diag}(\mathbf{S}(d))\right) \\
= & L_{c}(d)
\end{aligned}
$$


where $\mathbf{B}_{\infty}(d)$ is calculated given infinite past channel symbols. From (40), unlike [12] and [19], channel scattering function is not explicit but lies within $\mathbf{B}_{\infty}(d)$.

\section{Lower Bound $L_{c}(d)$ Over Ricean Fading Channels}

We extend our lower bound results to Ricean fading channels. Let $\rho$ denote a Ricean fading parameter which is the ratio of the fixed to a scatter part. $\rho$ is assumed independent of the transmission distance $d$ and identical for every delay path. The approximate CIR $h_{n, l}[0]$ of the $l$ th path is modeled as

$$
h_{n, l}[0]=\sigma_{l}\left(A_{l} e^{j \phi_{l}}+s_{n, l}\right)
$$

where

$$
\rho=\frac{A_{l}^{2}}{E\left[\left|s_{n, l}\right|^{2}\right]}
$$

and

$$
A_{l}^{2}=\frac{\rho}{\rho+1}, \quad E\left[\left|s_{n, l}\right|^{2}\right]=\frac{1}{\rho+1} .
$$

$E\left[\left|h_{n, l}[0]\right|^{2}\right]=\sigma_{l}^{2} \cdot \phi_{l}$ is assumed uniformly distributed from $-\pi$ to $\pi$ and uncorrelated across different delay paths. We follow the same approach to derive the lower bound as in Section III-B. For $h(\mathbf{Y})$, using (42), $H_{n, k}$ is

$$
H_{n, k}=\sum_{l=0}^{L-1} \sigma_{l} A_{l} e^{j \phi_{l}} e^{-j 2 \pi l k / K}+\sum_{l=0}^{L-1} \sigma_{l} s_{n, l} e^{-j 2 \pi l k / K} .
$$

From (45), sum of the scatter part follows $\mathcal{C N}(0,1 /(\rho+1))$. This causes $H_{n, k} \sim \mathcal{C N}\left(D_{k}, 1 /(\rho+1)\right)$ where $D_{k}=$ $\sum_{l=0}^{L-1} \sigma_{l} A_{l} e^{j \phi_{l}} e^{-j 2 \pi l k / K}$.

For $h(\mathbf{Y} \mid \mathbf{X})$, we assume that the receiver can successfully track the fixed part $A_{l} \exp \left(j \phi_{l}\right)$ and the autocorrelation function of the approximate CIR is

$$
E\left[h_{n, l}[0] h_{n^{\prime}, l^{\prime}}^{*}[0]\right]=\left(A_{l}^{2}+R_{s}\left[n-n^{\prime}, l\right]\right) \sigma_{l}^{2} \delta\left[l-l^{\prime}\right]
$$

where $R_{s}\left[n-n^{\prime}, l\right]=E\left[s_{n, l} s_{n^{\prime}, l}^{*}\right]$. Then, apply (46) to calculate $\mathbf{B}_{n}(d)$ and obtain $h(\mathbf{Y} \mid \mathbf{X})$.

\section{Simulation Results}

We examine the capacity upper bound $U_{c}(d)$ and lower bound $L_{c}(d)$ of OFDM systems over frequency-dependent UW fading channels as a function of the distance $d$ between the transmitter and the receiver. The UW fading channel $G_{n, k}$ is modeled by two parts: the attenuation $Q^{2}\left(d, f_{k}\right)$ and statistical $H_{n, k}$ parts. The attenuation part is explained in Section II-B1. For the statistical part of the UW channel, the delay profile of the scattering function is assumed exponentially decaying whose maximum delay spread $\tau_{m}$ is set where the first and last arrival paths have 10-dB power difference. In the Doppler profile of the scattering function, its range is determined by $f_{d}$, the $3-\mathrm{dB}$ bandwidth of the frequency response, and its shape will be discussed in Section IV-A. For $A\left(f_{k}\right)$, the shipping activity $s=0.5$, and wind speed $w=10 \mathrm{~m} / \mathrm{s}$. For the OFDM transmitter, OFDM symbols are transmitted at frequency beyond $1 \mathrm{KHz}$. Frequencies below $1 \mathrm{KHz}$ are excluded since the data rate would increase very slightly from those bands. Energy allocation across transmit bandwidth $B_{c}(d)$ is implemented using (34) subject to power constraint (33). $P=145 \mathrm{dBre} / \mu \mathrm{Pa}$ and Rayleigh fading are assumed unless stated otherwise.

1) Limitations Due to the ICI: Because of the attenuation $Q^{2}\left(d, f_{k}\right)$, the variance of the ICI $\sigma_{I}^{2}\left(d, f_{k}\right)$ is frequency dependent. Using an approach similar to [26], its variance at the $k$ th subcarrier is

$$
\sigma_{I}^{2}\left(d, f_{k}\right)=\frac{1}{K} \sum_{k^{\prime} \neq k} \sigma_{x}^{2}\left(d, f_{k^{\prime}}\right) Q^{2}\left(d, f_{k^{\prime}}\right) Z\left(k-k^{\prime}\right)
$$

where

$$
\begin{aligned}
& Z\left(k-k^{\prime}\right) \\
& \quad=\sum_{q=-(K-1)}^{K-1}\left(1-\frac{|q|}{K}\right) \sum_{l=0}^{L-1} R_{h_{0}}[q, l] e^{-j 2 \pi\left(k-k^{\prime}\right) q / K} .
\end{aligned}
$$

$R_{h_{0}}\left[m-m^{\prime}, l-l^{\prime}\right]=E\left[h_{0}[m, l] h_{0}^{*}\left[m^{\prime}, l^{\prime}\right]\right]$ and $\sigma_{x}^{2}\left(d, f_{k}\right)=$ $E\left[\left|X_{n, k}\right|^{2}\right]$ is the energy allocation to $k$ th subcarrier based on the physical properties available at the transmitter as mentioned in Section III.

Our model assumes the ICI variance (47) is negligible compared to that of the ambient noise $A\left(f_{k}\right)(29)$. In the simulation, we limit the ICI variance to be at least $3 \mathrm{~dB}$ lower than $A\left(f_{k}\right)$. From (47), the ICI variance depends on $Q^{2}\left(d, f_{k}\right), \sigma_{x}^{2}\left(f_{k}\right)$ and the ranges and shape of the scattering function. We focus on two types of the scattering function: AR-1 and uniform whose 3-dB bandwidth is equal to $\lambda_{d}$. Let $S_{1}[l, \lambda]$ and $S_{2}[l, \lambda]$ denote the AR-1 and uniform scattering functions of $h_{n, l}[0]$ given by

$$
\begin{aligned}
& S_{1}[l, \lambda]=\frac{\sigma_{l}^{2}}{\left|1-\alpha_{l} e^{-j 2 \pi \lambda}\right|^{2}}, \quad \lambda \in[-0.5,0.5] \\
& S_{2}[l, \lambda]= \begin{cases}\frac{\sigma_{l}^{2}}{\left(2 \lambda_{d}\right)}, & |\lambda| \leq \lambda_{d} \\
0, & \lambda_{d}<|\lambda| \leq 0.5 .\end{cases}
\end{aligned}
$$

$\alpha_{l}$ from (49) is obtained by solving

$$
\frac{1}{\left|1-\alpha_{l} e^{-j 2 \pi \lambda_{d}}\right|^{2}}=\frac{1}{2\left(1-\alpha_{l}\right)^{2}}
$$

Note that $\left(f_{d}, \tau_{m}\right)$ of $S_{c}(\tau, \nu)$ is related to $\left(L, \lambda_{d}\right)$ of $S_{h}[l, \lambda]$ by $L=\left\lceil\tau_{m} B_{c}(d)\right\rceil$ and $\lambda_{d}=f_{d} T_{b}$ where $B_{c}(d)$ is the signal bandwidth from (34). $S_{1}[l, \lambda]$ and $S_{2}[l, \lambda]$ represent severe and mild channel variations, respectively. These scattering functions are assumed unchanged over the transmission ranges of interest.

Fig. 3 displays variance of the ICI at their widest spread of both scattering functions when $d=5 \mathrm{~km}$ such that its variance is at least $3 \mathrm{~dB}$ lower than that of the ambient noise for most of the transmission bandwidth. For the AR-1 model, $\tau_{m}=1 \mathrm{~ms}$ and $f_{d}=1 \mathrm{~Hz}$. For the uniform model, $\tau_{m}=5 \mathrm{~ms}$ and $f_{d}=$ $7 \mathrm{~Hz}$. These $\left(f_{d}, \tau_{m}\right)$ give the maximum channel variations that can be used in our derivation described in Section III when $d=$ $5 \mathrm{~km}$. Since the ICI is lower at longer distances due to stronger attenuation, these $\left(f_{d}, \tau_{m}\right)$ will be used as channel variation limitations for $d \geq 5 \mathrm{~km}$. Notice that $S_{2}[l, \lambda]$ occupy wider ranges of $\left(f_{d}, \tau_{m}\right)$ compared to $S_{1}[l, \lambda]$. This is due to the more severe impact on the ICI of the AR-1 model than the uniform model.

We notice that the 3-dB gap is violated when signal bandwidth is greater than $31 \mathrm{kHz}$. These account for only $0.39 \%$ of the total signal energy and have negligible impact on the capacity as justified in Appendix I. 


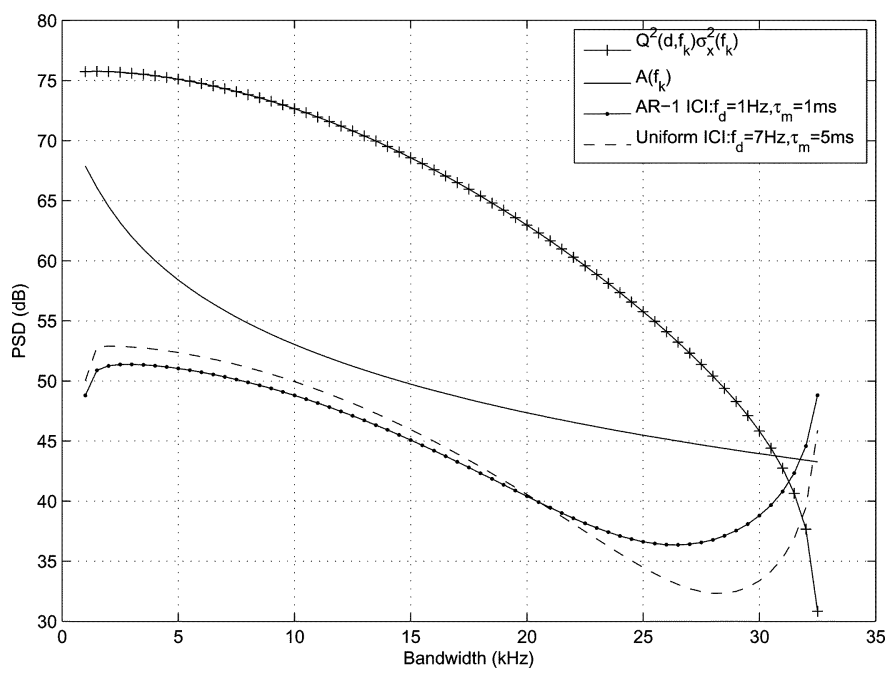

Fig. 3. PSD of the received signal $\left(Q^{2}\left(d, f_{k}\right) \sigma_{x}^{2}\left(f_{k}\right)\right)$, ambient noise $\left(A\left(f_{k}\right)\right)$, and the ICI variance of the AR-1 and uniform scattering functions at $d=5 \mathrm{~km}$.

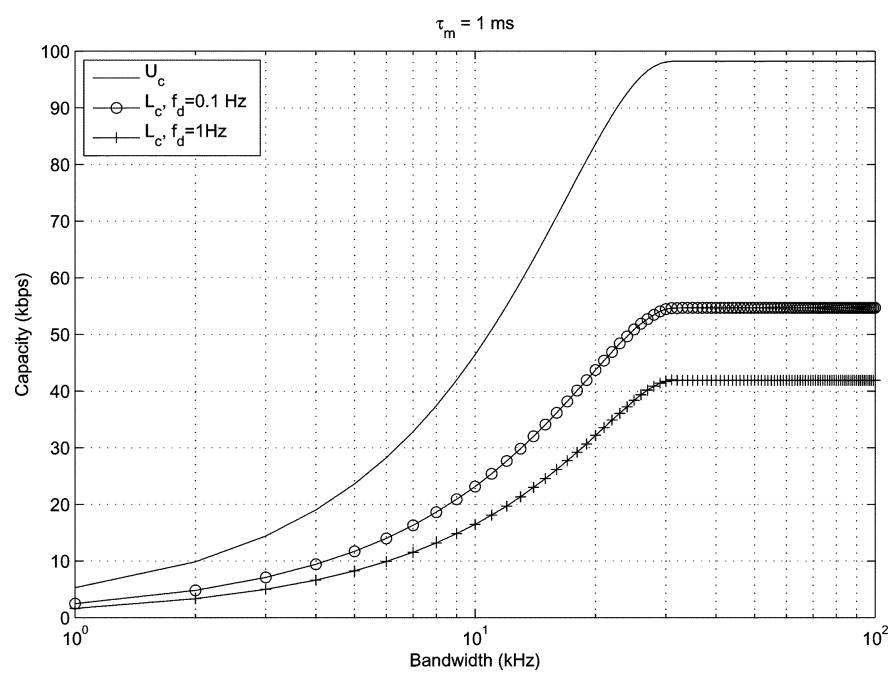

Fig. 4. $U_{c}(d)$ and $L_{c}(d)$ versus bandwidth when $d=5 \mathrm{~km}$ for AR-1 model.

Under these limits, the subcarrier spacing $F$ and OFDM symbol interval $T_{b}$ are determined as follows. For the AR-1, $F=1 / \tau_{m} / 2=500 \mathrm{~Hz}$ and $T_{b}=(1 / F)+\tau_{m}=15 \mathrm{~ms}$. For the uniform model, $F=100 \mathrm{~Hz}$ and $T_{b}=3 \mathrm{~ms}$. This guarantees that $F$ is less than the channel coherence bandwidth $\left(1 / \tau_{m} / 2\right)$ with no IBI.

2) Impact of the Signal Bandwidth: Fig. 4 shows the impact of the signal bandwidth $B$ on $U_{c}(d)$ and $L_{c}(d)$ for $S_{1}[l, \lambda]$. From the figure, both $U_{c}(d)$ and $L_{c}(d)$ increase as a function of signal bandwidth $B$ and remain fixed when $B$ is greater than a certain value. We define this value as the capacity-maximizing bandwidth $B_{c}(d)$ which is a signal bandwidth that maximizes both $L_{c}(d)$ and $U_{c}(d)$. Both $L_{c}(d)$ and $U_{c}(d)$ remain fixed for $B>B_{c}(d)$ since no information is sent at frequencies beyond $B_{c}(d)$. The gap is rather wide due to the limited mutual information that can be conveyed by the PSK constellation.

3) Impact of the Ranges and Shape of the Scattering Function: Figs. 5 and 6 show the impact of the ranges of $\left(f_{d}, \tau_{m}\right)$ on $L_{c}(d)$ over the distance for $S_{1}[l, \lambda]$ and $S_{2}[l, \lambda]$, respectively. In both figures, the left column exhibits the impact of varying $f_{d}$ while the right column exhibits the impact of varying $\tau_{m}$. The upper row displays $U_{c}(d)$ and $L_{c}(d)$ as a function of the transmission distance. Also shown are their corresponding capacity maximizing bandwidth $B_{c}(d)$ and results from the AWGN channel [6]. The lower row displays the gap between $L_{c}(d)$ and $U_{c}(d)$, exhibited as the ratio of $L_{c}(d)$ to $U_{c}(d)$. As expected, the ratio between $L_{c}(d)$ and $U_{c}(d)$ increases as either $f_{d}$ or $\tau_{m}$ increases. The ratio could be as high as 0.42 as shown in Fig. 6 when $d=5 \mathrm{~km}$. This is due to the higher prediction error influenced by stronger channel variations.

We compare the impact of the shape of the scattering function in Fig. 7 when $f_{d}=1 \mathrm{~Hz}$ and $\tau_{m}=1 \mathrm{~ms}$. We set $F=500$ $\mathrm{kHz}$ and $T_{b}=15 \mathrm{~ms}$. From the figure, $L_{c}(d)$ from $S_{1}[l, \lambda]$ is lower than that of $S_{2}[l, \lambda]$ as shown in Fig. 7(a). From Fig. 7(b), the ratio $L_{c}(d) / U_{c}(d)$ for $S_{1}[l, \lambda]$ is approximately within $0.42-0.65$ while that of $S_{2}[l, \lambda]$ is more than 0.60 .

4) Impact of Over Ricean Fading Channels: Fig. 8 shows the upper and lower bounds to the channel capacity over Ricean fading channels. The Ricean fading parameter $\rho$ is set to $-5,0$, 5 , and $10 \mathrm{~dB}$, identical for every path and independent of the distance. The Doppler spread profile of the scatter part $\left[R_{s}[n, l]\right.$ from (46)] is assumed uniformly distributed. The fixed part is perfectly known at the receiver. From the figure, the gap between the upper and lower bounds decreases as $\rho$ increases which is due to the reduced power in the scatter part of the channel.

5) Impact of the Transmission Distance: From Figs. 5 and 6, both $U_{c}(d)$ and $L_{c}(d)$ decrease at longer distance owing to strong channel attenuation which determines $B_{c}(d)$. The ratio between $L_{c}(d)$ and $U_{c}(d)$ tends to increase at either short $(d \leq$ $5 \mathrm{~km})$ or very long $(d \geq 65 \mathrm{~km})$ distance. The gap at a short transmission distance is due to the energy wasted because of the PSK constellation while the gap at a very long distance is due to the higher prediction error because of the stronger attenuation.

6) Impact of the Transmit Power: Fig. 9 exhibits the impact of the transmit power $(P)$ on $L_{c}(d)$ [Fig. 9(a)] and $B_{c}(d)$ [Fig. 9(b)] for $S_{1}[l, \lambda] . f_{d}$ is $1 \mathrm{~Hz}$ and $\tau_{m}$ is $1 \mathrm{~ms} . U_{c}(d)$ is plotted as a reference. From the figure, a significant decrease in $L_{c}(d)$ and $B_{c}(d)$ occurs especially at the long distance. This shows that for data transmission at low power, a short distance or multiple short hops across the transducers are preferred to one long transmission.

\section{EXPERIMENTAL DATA}

We investigate the capacity of OFDM systems using the scattering function from real UW environments measured from the RACE08 experiment. We select data from the receiving arrays which are $1000 \mathrm{~m}$ from the transducer. The array is a 12-element vertical array with $12-\mathrm{cm}$ spacing between elements. 8-PSK signals are upsampled by a factor of ten and filtered by a square root raised-cosine filter with a rolloff factor 0.25 . A block of data which contain 64 data symbols are transmitted every $28.7 \mathrm{~ms}$. A guard period is inserted between blocks to avoid the IBI. The bandwidth is $4.8 \mathrm{kHz}$ at $12-\mathrm{kHz}$ carrier frequency. Fig. 10(a) shows a contour plot of the estimates of the scattering function and Fig. 10(b) shows their corresponding PDP of process I-IV obtained from four different measurement periods. The scattering function is estimated using the AR spectral estimator [31]. 

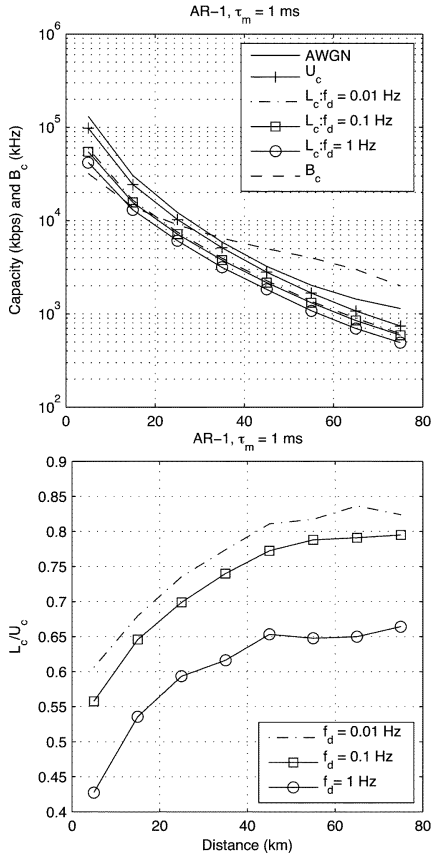

(a)
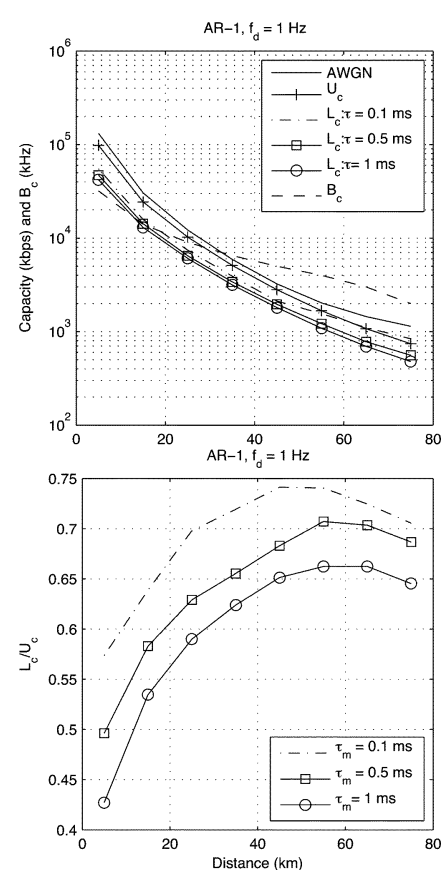

(b)

Fig. 5. Impact of the (a) Doppler spread $f_{d}$ (left column) and (b) delay spread $\tau_{m}$ (right column) on $L_{c}(d)$ versus the distance and its corresponding $L_{c}(d) / U_{c}(d)$ for the AR-1 scattering function.
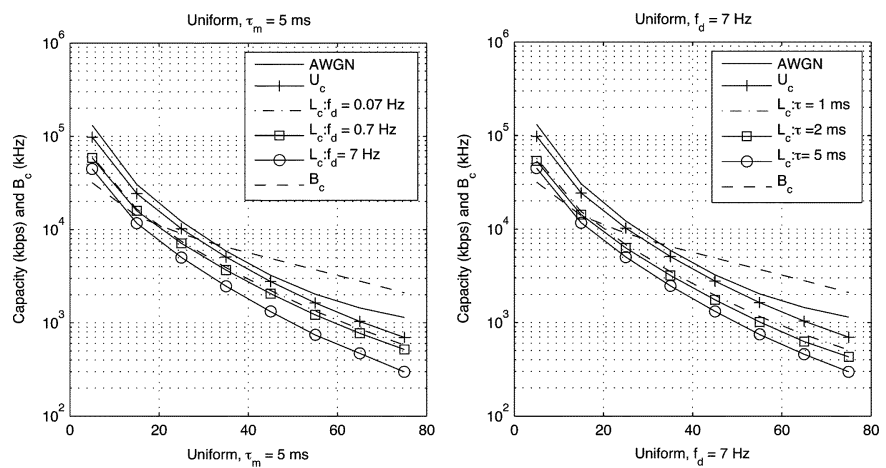

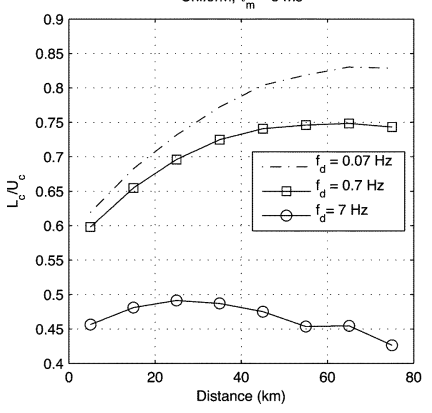

(a)

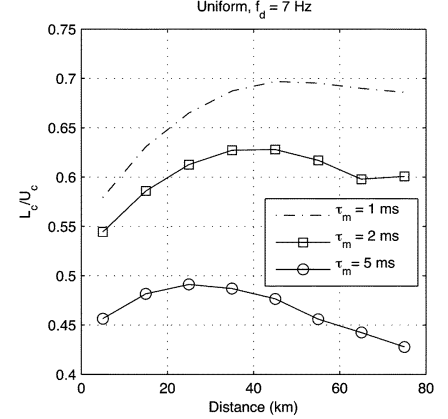

(b)

Fig. 6. Impact of the (a) Doppler spread $f_{d}$ (left column) and (b) delay spread $\tau_{m}$ (right column) on $L_{c}(d)$ versus the distance and its corresponding $L_{c}(d) / U_{c}(d)$ for the uniform Doppler spread profile.

The sampling frequency in delay domain is $3.9 \mathrm{kHz}$ and $35 \mathrm{~Hz}$ $\left(1 / 28.7 \times 10^{3}\right)$ in the absolute time domain.

As shown in (22) in Section II-E, frequency-dependent UW fading channels consist of two parts: the attenuation and statistical parts. In this section, we use the estimate scattering function from RACE08 experiments to approximate the statistical

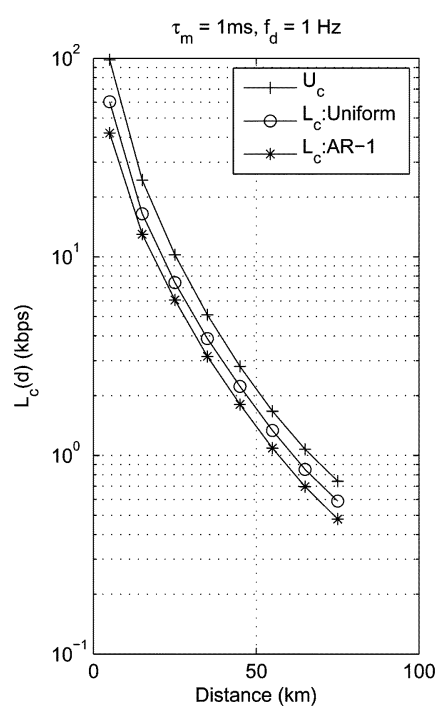

(a)

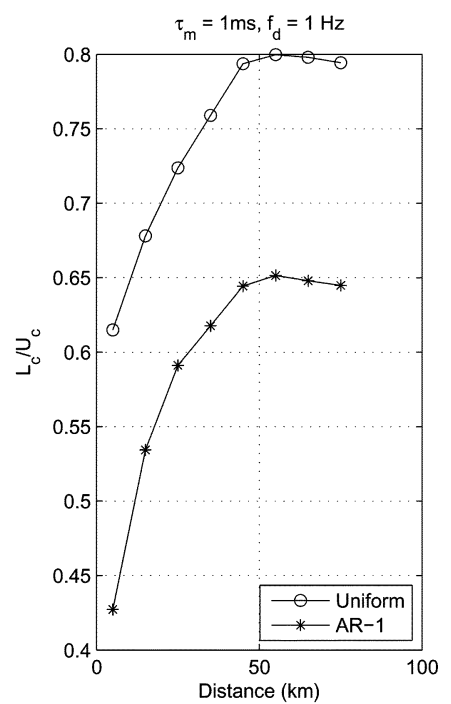

(b)
Fig. 7. Impact of the shape of the scattering function on (a) $L_{c}(d)$ and (b) $L_{c}(d) / U_{c}(d)$.

part of the UW channel, i.e., the scattering function. The frequency selectivity due to the attenuation on the estimate scattering function is negligible because of small transmit bandwidth $(4.8 \mathrm{kHz})$. The approximation of the estimate scattering function is divided into two parts: the PDP and Doppler spectrum.

1) PDP: Based on the observations sampled at $3.9 \mathrm{kHz}$, we fit the PDP using linear interpolation. The PDP ends when the power difference between the first and last paths is less than -10 $\mathrm{dB}$ as confirmed by the experiment.

2) Doppler Spectrum: We model channel variation of each estimate tap delay (sampled at $3.9 \mathrm{kHz}$ ) by the AR- $q_{l}$ process whose order $q_{l}$ is calculated using the AIC [31] method. Note that data obtained in the absolute time domain are sampled at $35 \mathrm{~Hz}$. Let $q_{l}$ and $\mathbf{a}_{l}=\left[a_{1} \cdots a_{q_{l}}\right]$ denote the AR- $q_{l}$ order and predictor coefficients of the estimate $l$ th delay path. We approximate the arrival paths which arrive between the estimate $l$ th and $(l+1)$ th delay paths to share the same AR- $q_{l}$ order and $\mathbf{a}_{l}$ predictor coefficients with the $l$ th path.

We calculate $U_{c}(d)$ and $L_{c}(d)$ of the OFDM systems under UW channels whose statistical properties are approximated from RACE08 experiment. These approximate statistical properties are assumed unchanged over the transmission range of interest. The attenuation and the ambient noise PSD are modeled using the same parameters as mentioned in Section IV. Power is set at $145 \mathrm{dBre} / \mu \mathrm{Pa}$ and energy allocation across the bandwidth $B_{c}(d)$ is calculated using (34). Fig. 11(a) shows $L_{c}(d)$ and $U_{c}(d)$ from process I-IV over a range of the distance. Their corresponding $L_{c} / U_{c}$ are displayed in Fig. 11(b). From the results, process II yields the best performance while process IV yields the worst. This is due to high Doppler spread at the dominant arrival paths in process IV while process II experiences smallest Doppler spread for almost every arrival path as shown in Fig. 10. Processes I and III exhibit similar results although process III is slightly worse since more dominant paths experience stronger Doppler spread compared to process I. 


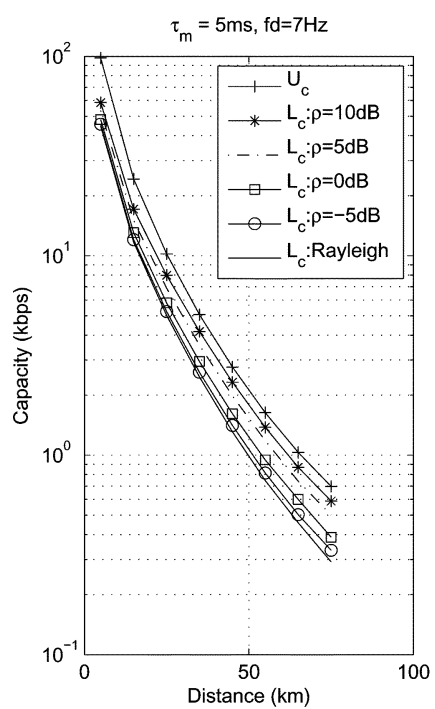

(a)

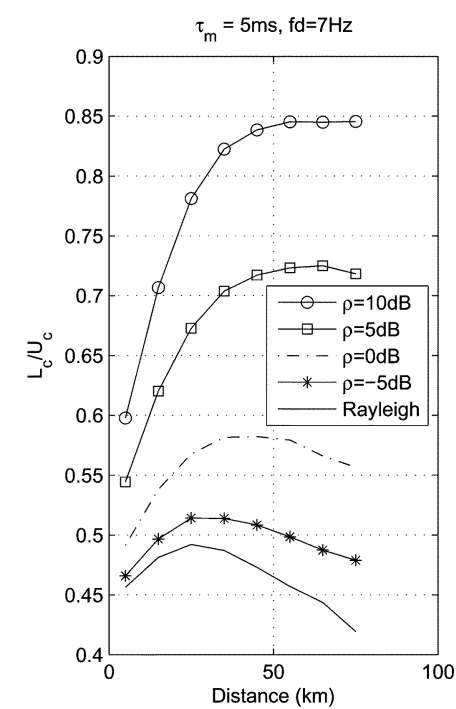

(b)
Fig. 8. Upper and lower bounds to the channel capacity over (a) Ricean fading channels and (b) its corresponding $L_{c}(d) / U_{c}(d) . f_{d}=7 \mathrm{~Hz}$ and $\tau_{m}=5 \mathrm{~ms}$ with uniform Doppler spread profile.

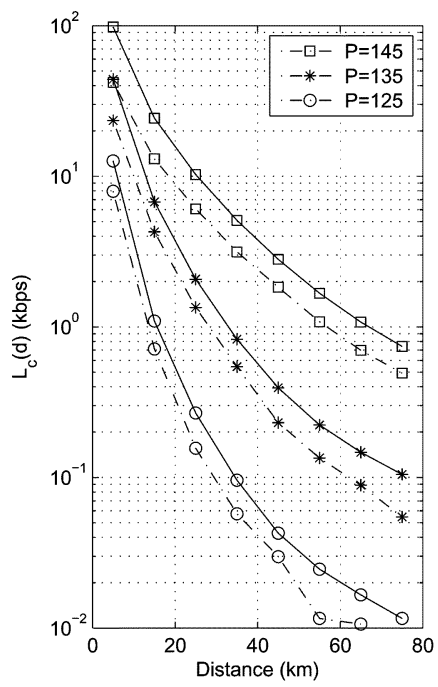

(a)

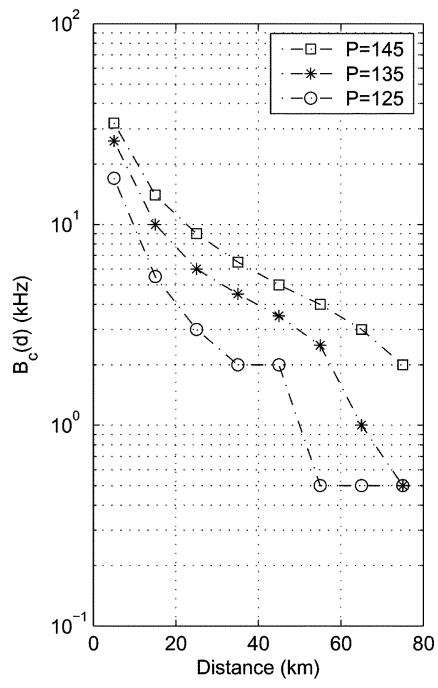

(b)
Fig. 9. Impact of the transmit power $(P)$ on (a) $L_{c}(d)$ and (b) $B_{c}(d)$.

\section{APPENDIX I \\ ICI JUSTIFICATION}

To investigate the ICI impact, we ran a simulation assuming that the ICI behaves as an independent complex Gaussian RV. Therefore, the total noise accumulated in the simulation is the ICI plus the ambient noise. From (1), by including the ICI, the received signal $Y_{n, k}$ can be written as

$$
\begin{aligned}
Y_{n, k} & =G_{n, k}(d) X_{n, k}+C_{n, k}+N_{n, k} \\
& =G_{n, k}(d) X_{n, k}+Z_{n, k}
\end{aligned}
$$

where $Z_{n, k}$ is the complex Gaussian noise consisting of the ambient and ICI noise whose variance is equal to $E\left[\left|C_{n, k}\right|^{2}\right]+$ $A\left(f_{k}\right)$. Using this assumption, we calculate the mutual information and present the upper $\left(U_{c}(d)\right)$ and lower $\left(L_{c}(d)\right)$ bounds as a function of bandwidth in Fig. 12. The distance between the
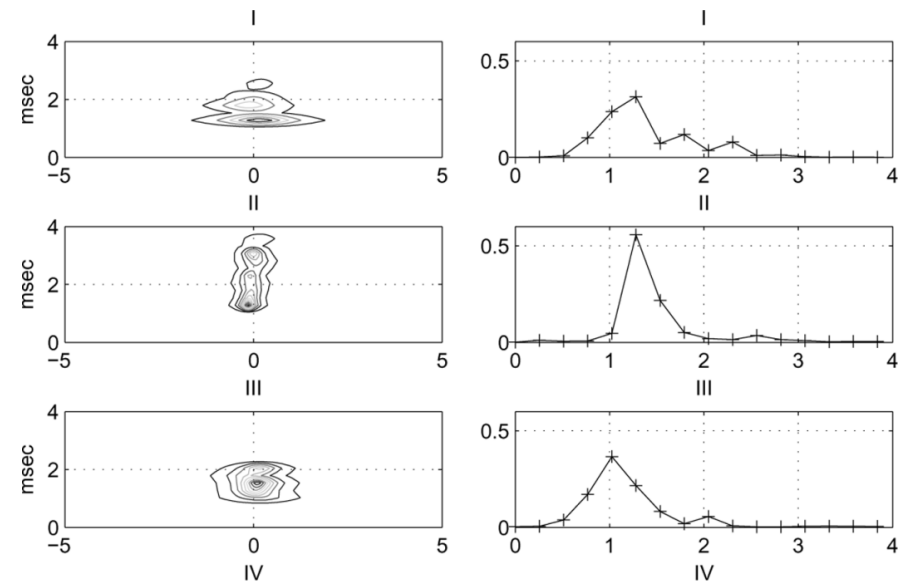

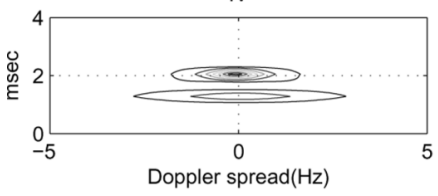

(a)

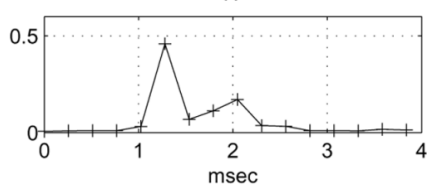

(b)
Fig. 10. (a) The scattering function estimates and (b) their corresponding normalized PDP from four different measurement periods.

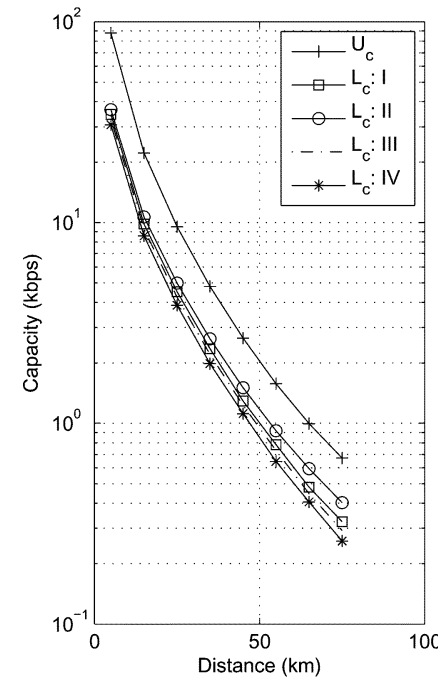

(a)

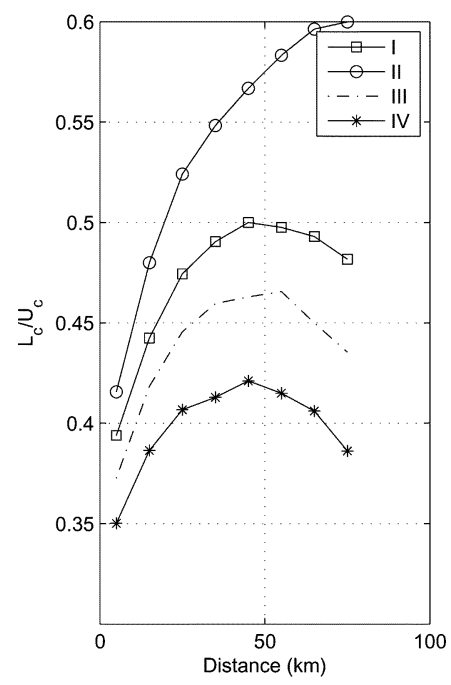

(b)
Fig. 11. (a) $L_{c}(d), U_{c}(d)$ and (b) its corresponding $L_{c}(d) / U_{c}(d)$ over experimental UW fading channels.

transmitter and the receiver is $5 \mathrm{~km}$. This distance gives highest ICI variance since longer distance means higher attenuation resulting in lower ICI. This figure can be compared with Fig. 4. From Fig. 12, when $f_{d}=1 \mathrm{~Hz}$, because of the ICI, $U_{c}(d)$ is reduced by $5.89 \%$ and $L_{c}(d)$ is reduced by $3.03 \%$. Moreover, the ratio $L_{c}(d) / U_{c}(d)$ when taking into account the ICI is 0.439 while the ratio $L_{c}(d) / U_{c}(d)$ without the ICI is 0.426 . For $f_{d}=$ $0.1 \mathrm{~Hz}, U_{c}(d)$ is reduced by $0.68 \%$ due to the ICI while $L_{c}(d)$ is reduced by $0.47 \%$.

In conclusion, we have shown that by taking into account the ICI as an additive complex Gaussian noise, $U_{c}(d)$ is reduced by at most $5.89 \%$ while $L_{c}(d)$ is reduced by at most $3.03 \%$. This reduction is quite small and has little impact on the overall performance, and justifies our ICI setting. 


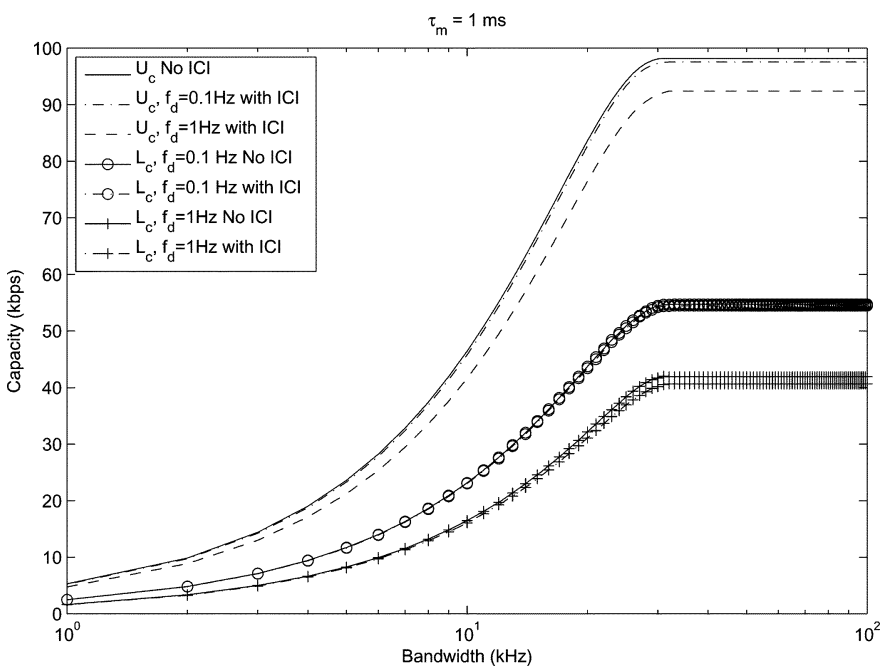

Fig. 12. Impact of the ICI on $U_{c}(d)$ and $L_{c}(d)$ versus bandwidth when $d=5$ $\mathrm{km}$ for AR-1 model.

APPENDIX II

\section{$I(\mathbf{Y} ; \mathbf{G}(d) \mid \mathbf{X})$ DERIVATION}

To calculate $I(\mathbf{Y} ; \mathbf{G}(d) \mid \mathbf{X})$, use the chain rule of the differential entropy [11]

$$
\begin{aligned}
& I(\mathbf{Y} ; \mathbf{G}(d) \mid \mathbf{X}) \\
& =h(\mathbf{Y} \mid \mathbf{X})-h(\mathbf{Y} \mid \mathbf{G}(d), \mathbf{X}) \\
& =h\left(\mathbf{Y}_{0}, \mathbf{Y}_{1}, \ldots, \mathbf{Y}_{N-1} \mid \mathbf{X}\right)-N \sum_{k=0}^{K-1} \log \left(\pi e A\left(f_{k}\right)\right) \\
& =\sum_{n=0}^{N-1} h\left(\mathbf{Y}_{n} \mid \mathbf{Y}_{0} \cdots \mathbf{Y}_{n-1}, \mathbf{X}\right)-N \sum_{k=0}^{K-1} \log \left(\pi e A\left(f_{k}\right)\right)
\end{aligned}
$$

where

$$
\begin{aligned}
& h\left(\mathbf{Y}_{n} \mid \mathbf{Y}_{0} \ldots \mathbf{Y}_{n-1}, \mathbf{X}\right) \\
& \quad \triangleq \log \left((\pi e)^{K} \operatorname{det}\left(\operatorname{Cov}\left[\mathbf{Y}_{n} \mid \mathbf{Y}_{0} \ldots \mathbf{Y}_{n-1}, \mathbf{X}\right]\right)\right) .
\end{aligned}
$$

To calculate $\operatorname{Cov}\left[\mathbf{Y}_{n} \mid \mathbf{Y}_{0} \cdots \mathbf{Y}_{n-1}, \mathbf{X}\right]$, we begin with the mean

$$
\begin{aligned}
& E\left[\mathbf{Y}_{n} \mid \mathbf{Y}_{0} \ldots \mathbf{Y}_{n-1}, \mathbf{X}\right] \\
& \quad=\operatorname{diag}\left(\mathbf{X}_{n}\right) E\left[\mathbf{G}_{n}(d) \mid \mathbf{Y}_{0}, \ldots, \mathbf{Y}_{n-1}, \mathbf{X}\right] \\
& \quad=\left(\operatorname{diag}\left(\mathbf{X}_{n}\right) \mathbf{Q}(d)\right) \hat{\mathbf{H}}_{n}(d)
\end{aligned}
$$

where (55) and (56) are obtained using $\mathbf{Y}_{n}=\operatorname{diag}\left(\mathbf{X}_{n}\right)$ $\mathbf{G}_{n}(d)+\mathbf{N}_{n}$ extracted from (2) and (27), respectively. $\widehat{\mathbf{H}}_{n}(d)=E\left[\mathbf{H}_{n}(d) \mid \mathbf{Y}_{0}, \ldots \mathbf{Y}_{n-1}, \mathbf{X}\right]$ is the MMSE channel estimate given the current and past detected symbols and can be written as the 1-step output of the linear $K \times K$ multiple-input-multiple-output (MIMO) predictor filter of length $J$

$$
\widehat{\mathbf{H}}_{n}(d)=\sum_{j=1}^{J} \mathbf{E}_{j}(d) \widetilde{\mathbf{H}}_{n-j}(d) .
$$

$\widetilde{\mathbf{H}}_{n}(d)=\left[\widetilde{H}_{n, 0}(d) \cdots \widetilde{H}_{n, K-1}(d)\right]^{T}$ and $\mathbf{E}_{j}(d)$ is the predictor coefficient of size $K \times K$. With OFDM input/output relationship (1), the observation $\widetilde{H}_{n, K-1}(d)$ is obtained by

$$
\widetilde{H}_{n, k}(d)=\frac{Y_{n, k}}{X_{n, k} Q\left(d, f_{k}\right)}=H_{n, k}+\frac{N_{n, k}}{X_{n, k} Q\left(d, f_{k}\right)} .
$$

Then, from (56), $\operatorname{Cov}\left[\mathbf{Y}_{n} \mid \mathbf{Y}_{0} \cdots \mathbf{Y}_{n-1}, \mathbf{X}\right]$ is

$$
\begin{aligned}
& \operatorname{Cov}\left[\mathbf{Y}_{n} \mid \mathbf{Y}_{0} \cdot s \mathbf{Y}_{n-1}, \mathbf{X}\right] \\
& \triangleq E\left[\left(\mathbf{Y}_{n}-\left(\operatorname{diag}\left(\mathbf{X}_{n}\right) \mathbf{Q}(d)\right) \widehat{\mathbf{H}}_{n}(d)\right)\right. \\
& \left.\quad \times\left(\mathbf{Y}_{n}-\left(\operatorname{diag}\left(\mathbf{X}_{n}\right) \mathbf{Q}(d)\right) \hat{\mathbf{H}}_{n}(d)\right)^{H}\right] \\
& =\left(\operatorname{diag}\left(\mathbf{X}_{n}\right) \mathbf{Q}(d)\right) \mathbf{B}_{n}(d)\left(\operatorname{diag}\left(\mathbf{X}_{n}^{*}\right) \mathbf{Q}(d)\right)+\operatorname{diag}\left(\mathbf{A}_{n}\right)
\end{aligned}
$$

where $\mathbf{B}_{n}(d)$ is the linear MMSE prediction error matrix obtained using the orthogonality principles $E\left[\left(\mathbf{H}_{\mathbf{n}}-\right.\right.$ $\left.\left.\widehat{\mathbf{H}}_{\mathbf{n}}(d)\right) \widetilde{\mathbf{H}}_{\mathbf{n}-\mathbf{j}}(d)\right]=\mathbf{0}$, which yields

$$
\begin{aligned}
\mathbf{B}_{n}(d) & =E\left[\left(\mathbf{H}_{n}-\widehat{\mathbf{H}}_{n}(d)\right)\left(\mathbf{H}_{n}-\widehat{\mathbf{H}}_{n}(d)\right)^{H}\right] \\
& =\mathbf{R}_{\mathbf{H}}(0)-\sum_{j=1}^{J} \mathbf{E}_{j}(d) \mathbf{R}_{\mathbf{H}}(-j)
\end{aligned}
$$

where the $(i, j)$ th entry of $\mathbf{R}_{\mathbf{H}}(m)$ is $R_{H}[m, i-j]=$ $E\left[H_{m^{\prime}+m, i} H_{m^{\prime}, j}^{*}\right]$. To solve $\mathbf{E}_{j}(d)$ and $\mathbf{B}_{n}(d)$, using the orthogonality principle $\mathbf{R}_{\mathbf{H}}(m)$ yields

$$
\begin{array}{r}
\mathbf{R}_{\mathbf{H}}(m)=\sum_{j=1}^{J} \mathbf{E}_{j}(d)\left(\mathbf{R}_{\mathbf{H}}(m-j)+\boldsymbol{\Gamma}_{V}(d) \delta(m-j)\right), \\
m=1, \ldots, J
\end{array}
$$

where $\boldsymbol{\Gamma}_{V}(d)=\operatorname{diag}\left(\mathbf{A}_{n}\right)\left(\mathbf{Q}(d) \mathbf{R}_{\mathbf{X}_{\mathbf{n}}}(d)\right)^{-1}$. Combining (61) and (60), the general form of the $J$ th-order multichannel normal equation is

$$
\boldsymbol{B n}_{\boldsymbol{n}}=\boldsymbol{R}_{\boldsymbol{H}} \boldsymbol{E}
$$

where $\boldsymbol{B}_{\boldsymbol{n}}=\left[\mathbf{B}_{n}(d)+\boldsymbol{\Gamma}_{V}(d), 0_{J}\right]^{T}, \boldsymbol{E}=\left[1,-\mathbf{E}_{1}(d)\right.$, $\left.\ldots,-\mathbf{E}_{J}(d)\right]^{T}$, and $\boldsymbol{R}_{\boldsymbol{H}}$ is a block-Toeplitz matrix which consists of $(J+1) \times(J+1)$ block entries of a $K \times K$ matrix $\mathbf{R}_{\mathbf{H}}(m)$. Solving (62) to obtain a set of the predictor coefficient $\mathbf{E}_{1}(d), \ldots, \mathbf{E}_{J}(d)$ and $\mathbf{B}_{n}(d)$ can be done using multichannel Levinson algorithm [31]. Note that from (60), $\mathbf{B}_{n}(d)$ depends on both the transmission $d$ and channel variations $R_{H}[m, k]$.

Substituting (59) into (54) and into (53) so that $I(\mathbf{Y} ; \mathbf{G}(d) \mid \mathbf{X})$ can be written as

$$
\begin{aligned}
& I(\mathbf{Y} ; \mathbf{G}(d) \mid \mathbf{X}) \\
& \quad=\sum_{n=0}^{N-1} \log \operatorname{det}\left(\boldsymbol{I}+\operatorname{diag}\left(\mathbf{X}_{n}\right) \mathbf{B}_{n}(d) \operatorname{diag}\left(\mathbf{X}_{n}^{*}\right) \operatorname{diag}\left(\mathbf{A}_{n}\right)^{-1}\right) \\
& =\sum_{n=0}^{N-1} \log \operatorname{det}\left(\boldsymbol{I}+\mathbf{B}_{n}(d) \operatorname{diag}\left(\mathbf{X}_{n}^{*}\right) \operatorname{diag}\left(\mathbf{A}_{n}\right)^{-1} \operatorname{diag}\left(\mathbf{X}_{n}\right)\right) \\
& \quad=\sum_{n=0}^{N-1} \log \operatorname{det}\left(\boldsymbol{I}+\mathbf{B}_{n}(d) \operatorname{diag}(\mathbf{S}(d))\right) .
\end{aligned}
$$

Equation (63) follows determinant identity $\operatorname{det}(\mathbf{I}+\mathbf{X Y})=$ $\operatorname{det}(\mathbf{I}+\mathbf{Y X})$. The $k$ th entry of the $K \times 1$ vector $\mathbf{S}(d)$ is $\sigma_{x}^{2}\left(d, f_{k}\right) Q^{2}\left(d, f_{k}\right) / A\left(f_{k}\right)$.

\section{ACKNOWLEDGMENT}

The authors would like to thank Prof. J. Preisig for providing the data from the RACE08 experiment. 


\section{REFERENCES}

[1] J. L. Cimini, "Analysis and simulation of a digital mobile channel using orthogonal frequency division multiplexing," IEEE Trans. Commun., vol. 33, no. 7, pp. 665-675, Jul. 1985.

[2] B. Li, S. Zhou, M. Stojanovic, L. Freitag, and P. Willett, "Multicarrier communication over underwater acoustic channels with nonuniform Doppler shifts," IEEE J. Ocean. Eng., vol. 33, no. 2, pp. 198-209, Apr. 2008.

[3] B. Li, S. Zhou, M. Stojanovic, L. Freitag, J. Huang, and P. Willett, "MIMO-OFDM over an underwater acoustic channel," in Proc. IEEE OCEANS, Sep. 2007, DOI: 10.1109/OCEANS.2007.4449296.

[4] B.-C. Kim and I.-T. Lu, "Parameter study of OFDM underwater communications system," in Proc. MTS/IEEE OCEANS Conf. Exhib., 2000, vol. 2, pp. 1251-1255.

[5] M. Stojanovic, "OFDM for underwater acoustic communications: Adaptive synchronization and sparse channel estimation," in Proc. IEEE Int. Conf. Acoust. Speech Signal Process., Apr. 4, 2008, pp. $5288-5291$.

[6] M. Stojanovic, "On the relationship between capacity and distance in an underwater acoustic channel," in Proc. IEEE 1st ACM Int. Workshop Underwater Netw., Los Angeles, CA, Sep. 2006, pp. 41-47.

[7] M. Stojanovic, "Underwater acoustic communications: Design considerations on the physical layer," in Proc. 5th Annu. Conf. Wireless Demand Netw. Syst. Services, Jan. 2008, DOI: 10.1109/WONS.2008. 4459349.

[8] D. Kilfoyle and A. Baggeroer, "The state of the art in underwater acoustic telemetry," IEEE J. Ocean. Eng., vol. 25, no. 1, pp. 4-27, Jan. 2000.

[9] T. Eggen, A. Baggeroer, and J. Preisig, "Communication over Doppler spread channels - Part I: Channel and receiver presentation," IEEE J. Ocean. Eng., vol. 25, no. 1, pp. 62-71, Jan. 2000.

[10] S. Dessalermos, "Undersea acoustic propagation channel estimation," M.S. thesis, Electr. Comput. Eng. Dept., Naval Postgrad. School, Monterey, CA, 2005.

[11] T. M. Cover and J. A. Thomas, Elements of Information Theory. New York: Wiley, 1991, ch. 2 and 9.

[12] D. Schafhuber, H. Boleskei, and G. Matz, "System capacity of wideband OFDM communications over fading channels without channel knowledge," in Proc. Int. Symp. Inf. Theory, 2004, pp. 389-394.

[13] A. Viterbi, "Performance of an M-ary orthogonal communication system using stationary stochastic signals," IEEE Trans. Inf. Theory, vol. IT-13, no. 3, pp. 414-422, Jul. 1967.

[14] M. Medard and R. Gallager, "Bandwidth scaling for fading multipath channels," IEEE Trans. Inf. Theory, vol. 48, no. 4, pp. 840-852, Apr. 2002.

[15] G. Durisi, H. Bolcskei, and S. Shamai, "Capacity of underspread noncoherent WSSUS fading channels under peak signal constraints," in Proc. IEEE Int. Symp. Inf. Theory, Jun. 2007, pp. 156-160.

[16] H. Kwon and T. Birdsall, "Channel capacity in bits per joule," IEEE J. Ocean. Eng., vol. 11, no. 1, pp. 97-99, Jan. 1986.

[17] T. J. Hayward and T. C. Yang, "Single- and multi-channel underwater acoustic communication channel capacity: A computational study," $J$. Acoust. Soc. Amer., vol. 122, pp. 1652-1661, Sep. 2007.

[18] G. Matz, "Characterization of non-WSSUS fading dispersive channels," in Proc. IEEE Int. Conf. Commun., May 2003, vol. 4, pp. 2480-2484.

[19] G. Durisi, H. Bolcskei, and S. Shamai, "Capacity of underspread WSSUS fading channels in the wideband regime," in Proc. IEEE Int. Symp. Inf. Theory, Jul. 2006, pp. 1500-1504.

[20] Y. Li and J. L. J. Cimini, "Bounds on the interchannel interference of OFDM in time-varying impairments," IEEE Trans. Commun., vol. 49, no. 3, pp. 401-404, Mar. 2001.

[21] D. Schafhuber, G. Matz, and F. Hlawatsch, "Pulse-shaping OFDM/ BFDM systems for time-varying channels: ISI/ICI analysis, optimal pulse design, and efficient implementation," in Proc. 13th IEEE Int. Symp. Pers. Indoor Mobile Radio Commun., Sep. 2002, vol. 3, pp. 1012-1016.

[22] S. Das and P. Schniter, "Max-SINR ISI/ICI-shaping multicarrier communication over the doubly dispersive channel," IEEE Trans. Signal Process., vol. 55, no. 12, pp. 5782-5795, Dec. 2007.

[23] C. S. Clay and H. Medwin, Acoustical Oceanography: Principles and Applications. New York: Wiley, 1977, ch. 3.

[24] J. G. Proakis, Digital Communications, 3rd ed. Boston, MA: McGraw-Hill, 1995, ch. 14.

[25] P. Bello, "Characterization of randomly time-variant linear channels," IEEE Trans. Commun. Syst., vol. 11, no. 4, pp. 360-393, Dec. 1963.
[26] M. Russell and G. Stuber, "Interchannel interference analysis of OFDM in a mobile environment," in Proc. IEEE 45th Veh. Technol. Conf., Jul. 1995, vol. 2, pp. 820-824.

[27] I. M. Telatar, "Capacity of multi-antenna Gaussian channels," Eur. Trans. Telecommun., vol. 10, no. 6, pp. 585-595, Nov./Dec. 1999.

[28] R. Iqbal, P. Sadeghi, and T. Abhayapala, "Constant power signaling in Rayleigh fading channels: Joint output probability distribution and information rate bounds," Channels, vol. 4, pp. 12-12, 2009.

[29] G. Durisi, U. Schuster, H. Bolcskei, and S. Shamai, "Noncoherent capacity of underspread fading channels," IEEE Trans. Inf. Theory, vol. 56, no. 1, pp. 367-395, 2010.

[30] A. Lozano, A. Tulino, and S. Verdii, "Optimum power allocation for multiuser OFDM with arbitrary signal constellations," IEEE Trans. Commun. Syst., vol. 56, no. 5, pp. 828-837, May 2008.

[31] S. L. Marple, Digital Spectral Analysis With Applications. Englewood Cliffs, NJ: Prentice-Hall, 1987.

[32] D. Schafhuber and G. Matz, "MMSE and adaptive prediction of timevarying channels for OFDM systems," IEEE Trans. Wireless Commun., vol. 4, no. 2, pp. 593-602, Mar. 2005.

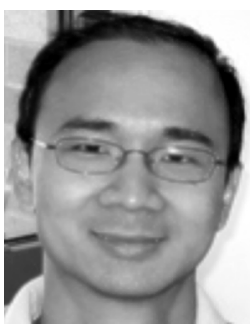

Chantri Polprasert (S'01-M'07) received the B.S. degree in electrical engineering from Chulalongkorn University, Bangkok, Thailand, in 1999, the M.S. degree in telecommunications from Asian Institute of Technology (AIT), Bangkok, Thailand, in 2000, and the Ph.D. degree in electrical engineering from the University of Washington, Seattle, in 2009.

Since 2010, he has been a Researcher at the National Electronics and Computer Technology Center, Wireless Innovation and Security Lab, Pathumthani, Thailand. His research interests include communications over time- and frequency-selective fading channels, channel estimation and equalization, and acoustical signal processing.

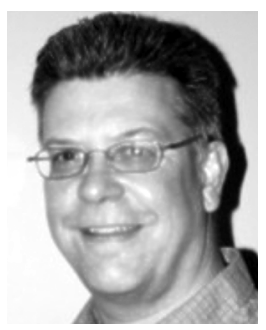

James A. Ritcey (S'74-M'83-SM'10-F'11) received the B.S.E. degree from Duke University, Durham, NC, the M.S.E.E. degree from Syracuse University, Syracuse, NY, and the Ph.D. degree in electrical engineering (communication theory and systems) from the University of California San Diego, San Diego, in 1985.

Since 1985, he has been with the Department of Electrical Engineering, University of Washington, Seattle, where he now holds the rank of Professor. His research interests include communications and statistical signal processing for radar and underwater acoustics.

Prof. Ritcey served as the General Chair of the 1995 International Conference on Communications in Seattle. He also served as Technical Program Chair of the 1992 and General Chair of the 1994 Asilomar Conference on Signals, Systems, and Computers and is currently a member of the Steering Committee.

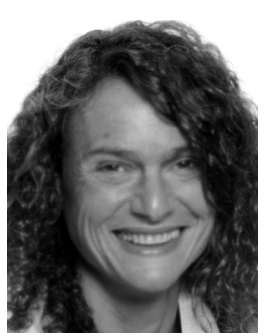

Milica Stojanovic (S'90-M'93-SM'08-F'10) graduated from the University of Belgrade, Belgrade, Serbia, in 1988, and received the M.S. and Ph.D. degrees in electrical engineering from Northeastern University, Boston, MA, in 1991 and 1993, respectively.

After a number of years with the Massachusetts Institute of Technology (MIT), Cambridge, where she was a Principal Scientist, she joined the faculty of Electrical and Computer Engineering Department, Northeastern University, in 2008. She is also a Guest Investigator at the Woods Hole Oceanographic Institution, Woods Hole, MA, and a Visiting Scientist at MIT. Her research interests include digital communications theory, statistical signal processing and wireless networks, and their applications to underwater acoustic communication systems.

Prof. Stojanovic is an Associate Editor for the IEEE Journal OF OCEANIC ENGINEERING and the IEEE TRANSACTIONS ON SIGNAL PROCESSING. 